\title{
UPPER BOUNDS FOR THE COVERING NUMBER OF CENTRALLY SYMMETRIC CONVEX BODIES IN $\mathbb{R}^{n}$
}

\begin{abstract}
SENLIN WU
Abstract. The covering number $c(K)$ of a convex body $K$ is the least number of smaller homothetic copies of $K$ needed to cover $K$. We provide new upper bounds for $c(K)$ when $K$ is centrally symmetric by introducing and studying the generalized $\alpha$-blocking number $\beta_{2}^{\alpha}(K)$ of $K$. It is shown that when a centrally symmetric convex body $K$ is sufficiently close to a centrally symmetric convex body $K^{\prime}$, then $c(K)$ is bounded by $\beta_{2}^{\alpha}\left(K^{\prime}\right)$ from above, where $\alpha$ is a properly chosen number. Related results in Minkowski geometry are also presented.
\end{abstract}

Mathematics subject classification (2010): 52A10, 46B20.

Keywords and phrases: Banach-Mazur distance, Birkhoff orthogonality, blocking number, covering number, generalized blocking number, Hadwiger's covering conjecture, radial projection of bisector, shadow boundary.

\section{REFERENCES}

[1] J. Alonso, H. Martini And Senlin Wu, On Birkhoff orthogonality and isosceles orthogonality in normed linear spaces, Aequat. Math., 83, 1-2 (2012), 153-189.

[2] K. BezDeK, The illumination conjecture and its extensions, Period. Math. Hungar., 53, 1-2 (2006), $59-69$.

[3] K. Bezdek, Classical Topics in Discrete Geometry, CMS Books in Mathematics/Ouvrages de Mathématiques de la SMC, Springer, New York, 2010.

[4] K. BEZDEK, Illuminating spindle convex bodies and minimizing the volume of spherical sets of constant width, Discrete Comput. Geom., 47, 2 (2012), 275-287.

[5] G. BIR KhofF, Orthogonality in linear metric spaces, Duke Math. J., 1, 2 (1935), 169-172.

[6] V. Boltyanski, Solution of the illumination problem for belt-bodies, Mat. Zametki, 58, 4 (1995), $505-511,638$.

[7] V. Boltyanski And H. Martini, Covering belt bodies by smaller homothetical copies, Beiträge Algebra Geom., 42, 2 (2001), 313-324.

[8] V. Boltyanski, H. Martini and P. S. Soltan, Excursions into Combinatorial Geometry, Universitext, Springer-Verlag, Berlin, 1997.

[9] K. BöröCZKy, JR., D. G. LARMAn, S. SEzGin, AND ChUANMing Zong, On generalized kissing numbers and blocking numbers, Rend. Circ. Mat. Palermo (2) Suppl., 65, part II (2000), 39-57.

[10] P. Brass, W. Moser And J. Pach, Research Problems in Discrete Geometry, Springer, New York, 2005.

[11] I. ŞERB, Rectangular modulus, Birkhoff orthogonality and characterization of inner product spaces, Commentationes Mathematicae Universitatis Carolinae, 40, 1 (1999), 107-119.

[12] L. Dalla, D. G. Larman, P. Mani-Levitska and Chuanming Zong, The blocking numbers of convex bodies, Discrete Comput. Geom., 24, 2-3 (2000), 267-277.

[13] J. GAO AND K. S. LAU, On the geometry of spheres in normed linear spaces, J. Austral. Math. Soc., 48, (1990), 101-112.

[14] I. Gohberg AND A. MAR Kus, A problem on covering of convex figures by similar figures, Izv. Mold. Fil. Akad. Nauk SSSR, 76, 10 (1960), 87-90.

[15] H. Hadwiger, Ungelöste probleme no. 20, Elem. Math., 12, (1957), 121. 
[16] Chan He And Yunan Cui, Some properties concerning Milman's moduli, J. Math. Anal. Appl., 329, (2007), 1260-1272.

[17] Á. G. HoRVÁth, On shadow boundaries of centrally symmetric convex bodies, Beiträge Algebra Geom., 50, 1 (2009), 219-233.

[18] R. C. JAMES, Orthogonality and linear functionals in normed linear spaces, Trans. Amer. Math. Soc., 61, (1947), 265-292.

[19] M. LASSAK, Solution of Hadwiger's covering problem for centrally symmetric convex bodies in $E^{3}$, J. London Math. Soc., 30, 3 (1984), 501-511.

[20] H. MARTini, Shadow-boundaries of convex bodies, Discrete Math., 155, 1-3 (1996), 161-172.

[21] H. Martini AND V. Soltan, Combinatorial problems on the illumination of convex bodies, Aequationes Math., 57, 2-3 (1999), 121-152.

[22] H. Martini, K. J. Swanepoel And G. Weiss, The geometry of Minkowski spaces - a survey. Part I, Expo. Math., 19, (2001), 97-142.

[23] H. Martini And SenLin Wu, Radial projections of bisectors in Minkowski spaces, Extracta Math., 23, 1 (2008), 7-28.

[24] L. SÁnchez And A. Ullán, Some properties of Gurarii's modulus of convexity, Arch. Math. (Basel), 71, 5 (1998), 399-406.

[25] LONG YU, Blocking numbers and fixing numbers of convex bodies, Discrete Math., 309, 23-24 (2009), $6544-6554$.

[26] Long YU AND ChuANMing Zong, On the blocking number and the covering number of a convex body, Adv. Geom., 9, 1 (2009), 13-29.

[27] Chunming Zong, Some remarks concerning kissing numbers, blocking numbers and covering numbers, Period. Math. Hungar., 30, 3 (1995), 233-238.

[28] Chuanming Zong, A quantitative program for Hadwiger's covering conjecture, Sci. China Math., 53, 9 (2010), 2551-2560. 